\title{
Development of a High-Performance Wind Turbine Equipped with a Brimmed Diffuser Shroud*
}

\author{
By Yuji Ohya, ${ }^{1)}$ Takashi Karasudani ${ }^{1)}$ Akira SAKURAI ${ }^{2)}$ and Masahiro InOuE ${ }^{3)}$ \\ 1) Research Institute for Applied Mechanics, Kyushu University, Kasuga, Japan \\ ${ }^{2)}$ Department of Aeronautics and Astronautics, Kyushu University, Fukuoka, Japan \\ ${ }^{3)}$ Sasebo National College of Technology, Sasebo, Japan
}

(Received October 6th, 2004)

\begin{abstract}
We have developed a new wind turbine system that consists of a diffuser shroud with a broad-ring brim at the exit periphery and a wind turbine inside it. The brimmed-diffuser shroud plays the role of a device for collecting and accelerating the approaching wind. Emphasis is placed on positioning the brim at the exit of the diffuser shroud. Namely, the brim generates a very low-pressure region in the exit neighborhood of the diffuser by strong vortex formation and draws more mass flow to the wind turbine inside the diffuser shroud. To obtain a higher power output of the shrouded wind turbine, we have examined the optimal form for the brimmed diffuser, such as the diffuser open angle, brim height, hub ratio, centerbody length, inlet shroud shape and so on. As a result, a shrouded wind turbine equipped with a brimmed diffuser has been developed, and demonstrated power augmentation for a given turbine diameter and wind speed by a factor of about five compared to a standard (bare) wind turbine.
\end{abstract}

Key Words: Wind Energy, Wind Turbine, Brimmed Diffuser, Wind Tunnel Experiment

\section{Introduction}

For the application of an effective energy resource in the future, the limitation of fossil fuels is clear and the security of alternative energy sources is an important subject. Furthermore, as concerns for environmental issues (i.e., global warming, etc.) continue to grow, the development and application of renewable and clean new energy resources are strongly expected. Among others, wind energy technologies have developed rapidly, and are about to play a big role in a new energy field. However, in comparison with the overall demand for energy, the scale of wind power usage is still small; especially, the level of development in Japan is extremely small. As for the reasons, various causes are conceivable. For example, the limited local area suitable for wind power plants, the complex terrain compared to that in European or North American countries and the turbulent nature of the local wind are pointed out. Therefore, the introduction of a new wind power system that produces higher power output even in areas where lower wind speeds and complex wind patterns are expected is strongly desired.

Wind power generation is proportional to the wind speed cubed. Therefore, a large increase in output is brought about if it is possible to create even a slight increase in the velocity of the approaching wind to a wind turbine. If we can increase the wind speed by utilizing the fluid dynamic nature around a structure or topography, namely if we can concentrate the wind energy locally, the power output of a wind turbine can be increased substantially. Although there have been several studies of collecting wind energy for wind tur-

(C) 2006 The Japan Society for Aeronautical and Space Sciences

*Presented at 33rd Fluid Dynamics Conference, September 28th, 2001. bines reported so far, ${ }^{1-4)}$ it has not been an attractive research subject conventionally. Unique research that was carried out intensively in the past was the examination of a diffuser-augmented wind turbine by Gilbert et al., ${ }^{1)}$ Gilbert and Foreman, ${ }^{2)}$ and others around 1980. In their studies, there was a focus on concentrating wind energy in a diffuser with a large open angle, where a boundary layer controlled with several flow slots was employed to realize a flow that goes along the inside surface of the diffuser. Thus, the method of boundary layer control prevents pressure loss by flow separation and increases the mass flow inside the diffuser. Also, $\mathrm{Nagai}^{3)}$ investigated a venturi-type structure (i.e., the front portion is a nozzle and the rear portion is a diffuser) as a structure concentrating the wind energy, especially paying attention to the effect of the diffuser part. He analyzed the flow inside the venturi-type structure using a momentum theory. Recently, a group from Ashikaga Institute of Technology has also reported a wind turbine with a nozzle-type concentrator. ${ }^{5)}$ Although several other ideas have been reported so far, most of them do not appear to be reaching commercialization. ${ }^{6)}$

The present study, regarding the development of a new type of wind power system with high output, aims at determining how to collect wind energy efficiently and what kind of wind turbine can generate energy effectively from the wind. For this purpose, we have developed a diffuser-type structure that is capable of collecting and accelerating the approaching wind. Namely, we have devised a diffuser shroud with a brim that is able to increase the wind speed of approaching wind substantially by utilizing various flow characteristics (e.g., the generation of a very-low-pressure region by vortex formation, flow entrainment by vortices 
and so on) of the inner or peripheral flows of a diffuser shroud equipped with a brim. Furthermore, we placed a wind turbine inside the diffuser shroud equipped with a brim and evaluated the power output generated. As a result, the new shrouded wind turbine equipped with a brimmed diffuser demonstrated power augmentation for a given turbine diameter and wind speed by a factor of about five compared to a standard wind turbine.

\section{Experimental Method and Numerical Analysis}

\subsection{Wind tunnel experiment}

The large boundary-layer wind tunnel of the Research Institute for Applied Mechanics, Kyushu University, was used. It has a $3.6 \mathrm{~m}$ wide $\times 2 \mathrm{~m}$ high $\times 15 \mathrm{~m}$ long measurement section and the maximum wind velocity is $30 \mathrm{~m} / \mathrm{s}$. Various hollow-structure models of the pyramid or cone type were made of polystyrene, acrylic or aluminum plates. The models were hung supported by strings in the center of the wind tunnel section. The distributions of wind velocity $U$ and static pressure $p$ along the central axis or periphery of the hollow-structure model were measured with an I-type hot-wire and a static-pressure tube using a traversing system. The static-pressure coefficient is defined as $C_{\mathrm{p}}=$ $\left(p-p_{\infty}\right) /\left(0.5 \rho U_{\infty}^{2}\right)$; where, $U_{\infty}$ is the approaching wind speed, $\rho$ is the air density, and $p_{\infty}$ is the static pressure that corresponds to $U_{\infty}$ in a far upstream position. The experimental arrangement is shown in Fig. 1. The representative scale length of a model is the entrance width (or entrance diameter) $D$ of $12 \mathrm{~cm}$ to $60 \mathrm{~cm}$, the wind velocity is $U_{\infty}=$ $5 \mathrm{~m} / \mathrm{s}$, and the Reynolds number is $R e=4 \times 10^{4}-1.3 \times$ $10^{5}$. In the case of using a big hollow-structure model, paying attention to the blockage effect in the wind tunnel, we removed the walls of the ceiling and both sides ranging $6 \mathrm{~m}$ in the center portion of the measurement section. Namely, we used a wind tunnel with an open-type test section to avoid a blockage effect. The smoke-wire technique was employed for the flow visualization experiment.

\subsection{Numerical method}

To understand the details of the flow passing through the diffuser shroud equipped with a brim, we performed direct numerical simulations (DNS) based on a finite-difference method. The governing equations are a continuity equation and Navier-Stokes equations for an incompressible viscous flow. The numerical analyses are made as two-dimensional calculations in this report to create easier calculations, although the wind-tunnel models are three-dimensional hollow bodies. As another study, we conducted an experiment using a two-dimensional diffuser equipped with a brim. The fundamental characteristics of the flow are similar to those of the three-dimensional diffuser with a brim. The numerical algorithm is based on the MAC method. To make a calculation with high accuracy, we employ a multi-directional finite-difference method ${ }^{7)}$ on the Cartesian coordinates. For the convective term, a third-order upwind scheme and a second-order central scheme for other spatial terms are used. For the time integration, the first-order Euler explicit method is used. The calculation grid is composed of a uniform interval rectangle grid of $601 \times 701$ and the spatial resolution is $\Delta x=\Delta y=0.02$ as the diffuser entrance width $D=1$. The calculation domain is $12 D \times 14 D$. The object form is approximated with rectangular grids.

\section{Development of a Collection-Acceleration Device for Wind (Diffuser Shroud Equipped with a Brim)}

\subsection{Selection of a diffuser-type structure as the basic form}

Since we have already reported that a diffuser-type structure is effective for collecting and accelerating the wind, ${ }^{8)}$ we summarize it briefly here. We examined the inner flow of three typical hollow structures, as shown in Fig. 2; namely a nozzle-type model that reduces the inside cross section, a cylindrical-type model that has a constant inside cross section and a diffuser-type model that expands the inside cross section downstream. For both ends of the hollow-structure model, the narrow-end is a square cross section of $D=12$ $\mathrm{cm}$ and the wide end is $D=24 \mathrm{~cm}$. For the nozzle and diffuser types, the angle of inclination $\phi$ is $3.7^{\circ}$. The length ratio $L / D$ is 7.7 ; here, $L$ is the model length.

Figures 3(a) and 3(b) show the wind velocity distribution $U / U_{\infty}$ and static pressure distribution $C_{\mathrm{p}}$ on the central axis of a hollow-structure model. As seen in Fig. 3(a), the diffuser model has a remarkable effect on the collection and acceleration of the approaching wind. A maximum of $U / U_{\infty} \sim$ 1.8 is shown in the neighborhood immediately after the entrance. Here, $x / L=0$ is the model entrance and $x / L=$ 1 is the model exit. Figures 4(a) and 4(b) show the flows inside and outside of the nozzle- and diffuser-type models. The flow is from left to right. As seen in the Fig. 4(a), the

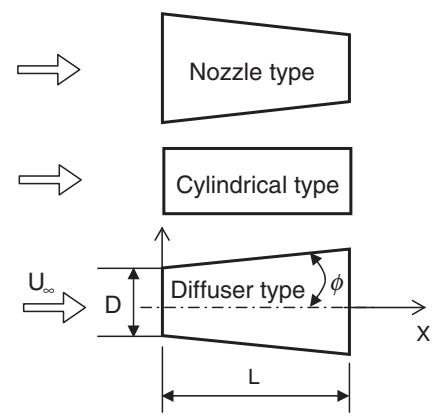

Fig. 1. Experimental arrangement.
Fig. 2. Three types of hollow structures 


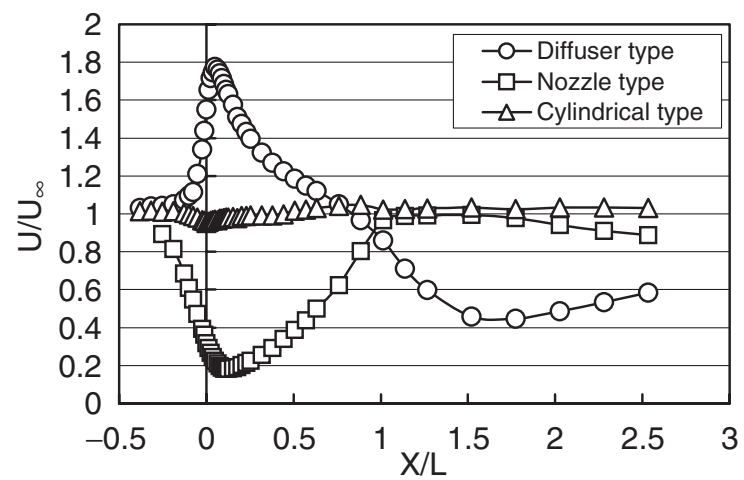

(a)

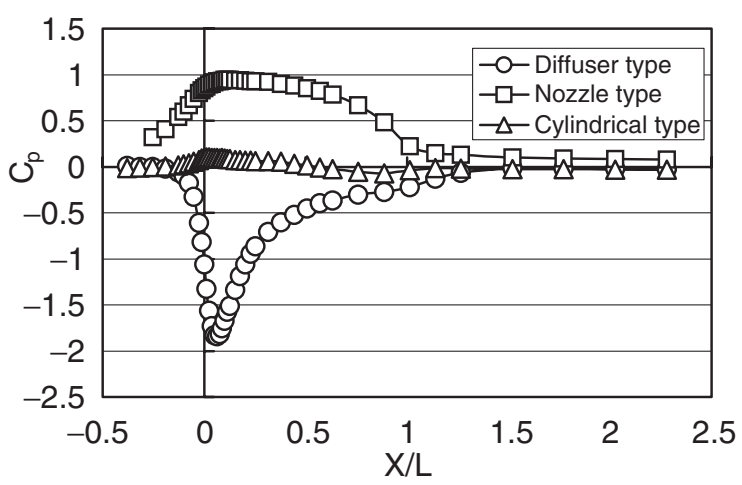

(b)

Fig. 3. (a) Wind velocity distribution on the central axis of a hollow structure, $L / D=7.7$. (b) Pressure distribution on the central axis of a hollow structure, $L / D=7.7$.

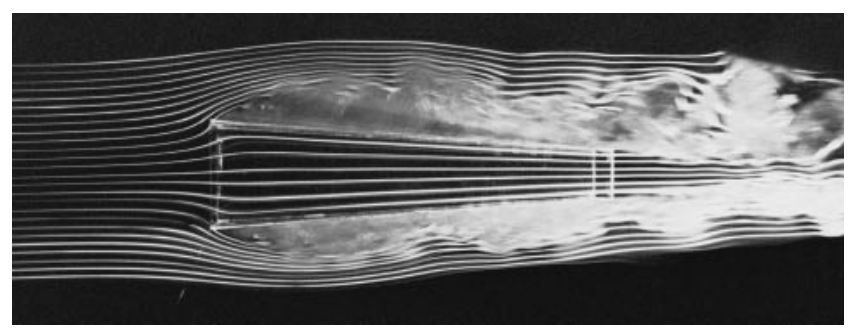

(a)

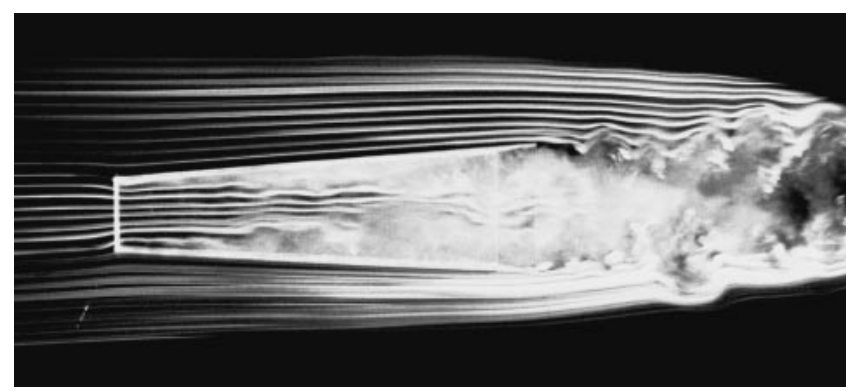

(b)

Fig. 4. (a) Flow around a nozzle-type model. (The smoke flows from left to right.) (b) Flow around a diffuser-type model. (The smoke flows from left to right.)

wind tends to avoid the nozzle-type model, while the wind flows into the diffuser-type model as it is inhaled, as seen in Fig. 4(b). Examining the optimal inclination angle $\phi$

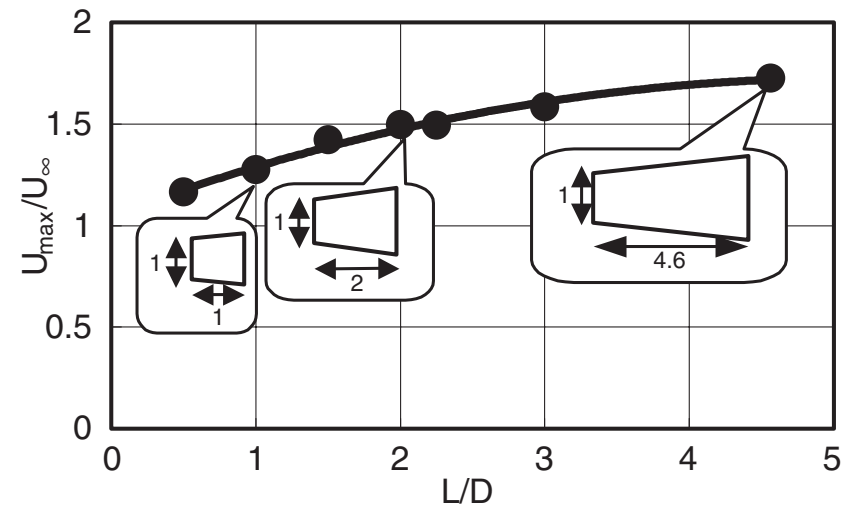

Fig. 5. Variation of the maximum wind velocity with the length of a diffuser-type model.

(the half-open angle) of this diffuser, it is found that the angle $\phi$ of around $4^{\circ}$ is most effective. Also, for the diffuser model, we examined the relationship between the maximum acceleration value $U_{\max } / U$ ( $U_{\max }$ is the maximum wind speed on the central axis) and the length ratio of $L / D$. Wind speed gradually increases with $L / D$, as shown in Fig. 5 . Thus, it was confirmed that the diffuser structure is most effective for collecting and accelerating the wind.

\subsection{Improvement of acceleration performance of the diffuser structure by the addition of periphery ap- pendages}

It was found that a remarkable increase in wind speed was obtained if a long diffuser body over $L / D=3$ is used, as shown in Fig. 5. However, in the case of commercialization it is preferable to have a short diffuser body with a $L / D$ of less than 2 that has similar performance to that of a long diffuser body. Therefore, we examined a short diffuser-type structure which is capable of providing more effective performance by applying various ideas to the short body. ${ }^{9)} \mathrm{A}$ diffuser model with a square section of $D=40 \mathrm{~cm}$ and a half-open angle of $\phi=4^{\circ}$ was used in this experiment.

As a result of several attempts, it was found that wind speed is increased by adding an appropriate entrance (called an inlet shroud) and a ring-type flange at the exit periphery (called a "brim", see Figs. 8, 9, 11 and 12) to the diffuser body. The inlet shroud opening has a smooth curved surface surrounding the entrance of the diffuser model. The brim is a ring-type square plate with a height of $h=10 \mathrm{~cm}(h / D=$ 0.25 ), and is attached vertically to the outer periphery of the diffuser model at the exit. As shown in Fig. 6, when both the inlet shroud and the brim are employed, a remarkable increase in wind speed can be obtained, exceeding the case of a diffuser model only, and achieving a high velocity that is 1.6 2.4 times greater than that of the approaching wind velocity $U_{\infty}$. For even a short diffuser $(L / D=1.5)$ with brim attached to the downstream edge, $U / U_{\infty} \sim 1.7$ was achieved.

The effect of the inlet shroud is found in the following point: It restrains flow separation at the entrance fairly well and the wind flows in more smoothly. Further examination of the inlet shroud was not conducted in the present experi- 


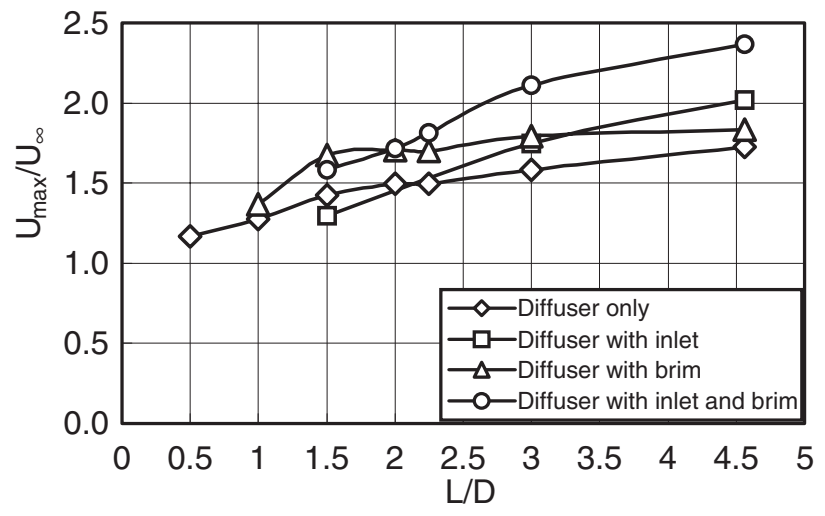

Fig. 6. Increase in the maximum wind velocity by the addition of an element to the diffuser-type model.

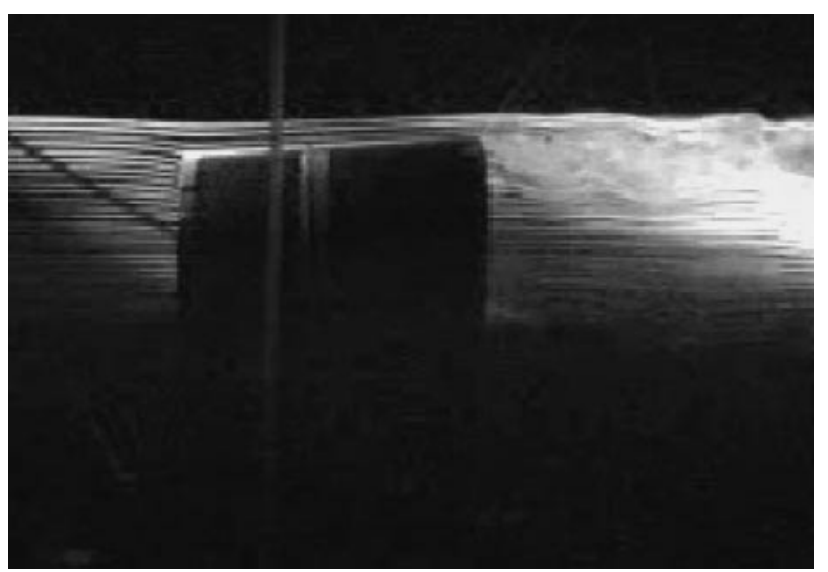

Fig. 7. Flow around a circular-diffuser model. (The smoke flows from the left to the right.)

ment. In the next section, we discuss the mechanism that causes the wind to increase when using a brim.

Concerning the aforementioned experiments, although we mainly examined acceleration performance using diffusers with square sections because of easier model production, we also examined diffusers with circular sections and found that they performed similar to that of the diffusers with square sections.

\subsection{Mechanism of wind velocity acceleration}

Here, we discuss the reason why the wind speed increases near the entrance when a brim is attached to the outer periphery of a diffuser exit. Figures 7 and 8 show the visualization results of both of the flows around a circular diffuser $(D=40 \mathrm{~cm})$ without a brim (Fig. 7) and one equipped with a brim (Fig. 8) using the smoke-wire technique. The brim used for the circular diffuser is a ring-type circular disk with a height of $h=10 \mathrm{~cm}(h / D=0.25)$ and is attached to the outer periphery of the diffuser exit. The flow is from left to right. In Fig. 7, clear vortex formation is not seen downstream of the diffuser without a brim, while vortex formation like the Karman vortex street is seen downstream of the brim in Fig. 8. As shown in Fig. 10(b) (discussed later), owing to the vortex formation, the static base pressure $p_{\mathrm{b}}$ in the exit area at around $x / L=1$ of the diffuser equipped with

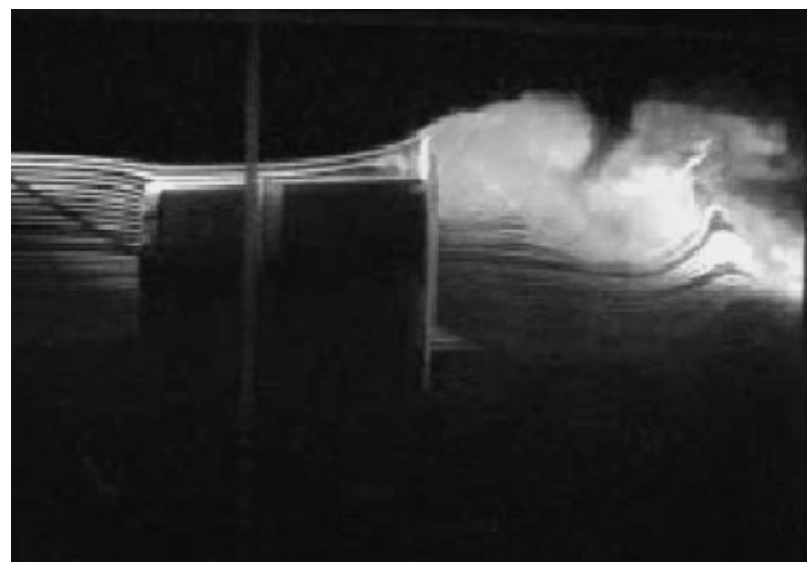

Fig. 8. Flow around a circular-diffuser model with a brim. (The smoke flows from left to right.)

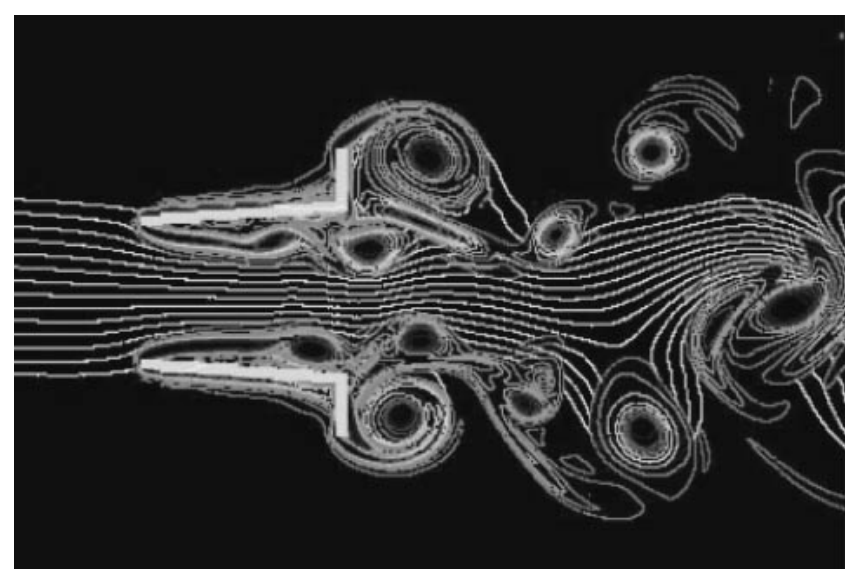

Fig. 9. Flow inside and outside a two-dimensional diffuser model with a brim using two-dimensional DNS.

The vorticity contours and streamlines are depicted.

a brim falls to a fairly low pressure compared to that of the upstream flow $p_{\infty}$, in comparison to the case of diffuser without a brim. The base-pressure coefficient at the exit of $x / L=1$ is defined as $C_{\mathrm{pb}}=\left(p_{\mathrm{b}}-p_{\infty}\right) /\left(0.5 \rho U_{\infty}^{2}\right)$. For the diffuser without a brim, $C_{\mathrm{pb}}=-0.2$. In contrast, for the diffuser equipped with a brim of $h / D=0.25, C_{\mathrm{pb}}=$ -0.75 .

Figure 9 shows the results of a two-dimensional DNS. The Reynolds number based on diffuser entrance width $D$ is $3 \times 10^{4}$. From the vorticity contours and streamlines in Fig. 9, we can see the flow behavior inside and outside the brimmed diffuser. Strong vortex formation behind the brim is clearly observed in the two-dimensional calculation; especially, the strong vortex formation outside the brim. In the flow inside the diffuser, a separated shear layer at the entrance grows to a vortex near the exit and is entrained into the region behind the brim. Also, from the smoke streaklines in Fig. 8 and from the streamlines in Fig. 9, we can see that the approaching flow is inhaled into the diffuser near the entrance. Thus, the brim generates a very low-pressure region in the near wake of the diffuser owing to the formation of a strong vortex and draws the flow into the diffuser. 


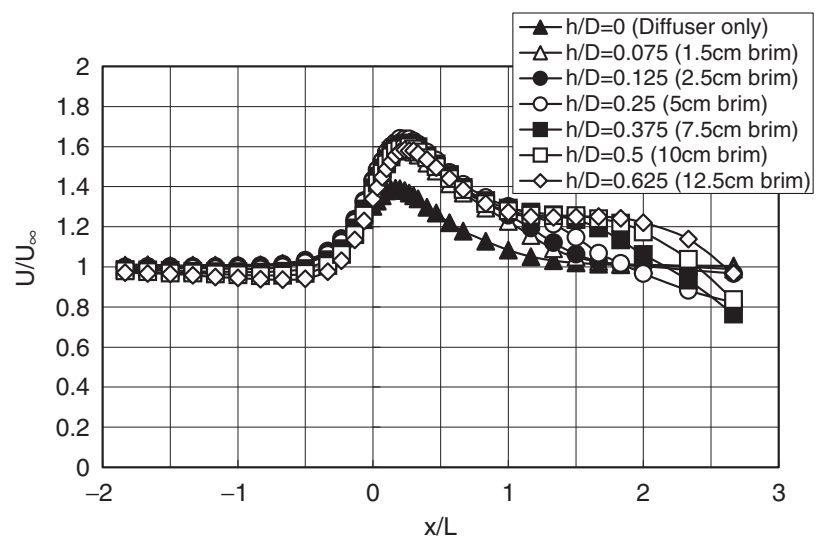

(a)

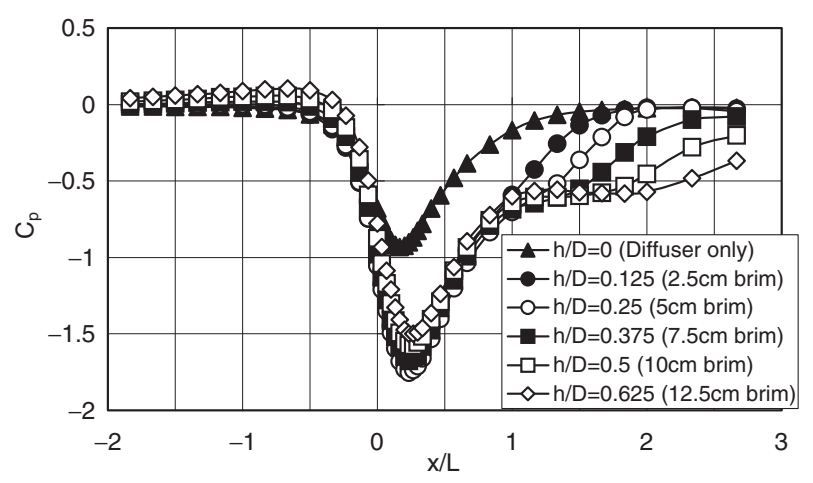

(b)

Fig. 10. (a) Wind velocity distribution on the central axis of a circulardiffuser model with and without a brim, $L / D=1.5$. (b) Static pressure distribution on the central axis of a circular-diffuser model with and without a brim, $L / D=1.5$.

\subsection{Optimum size of the brim}

We examined the optimum size of the brim to obtain the largest increase in wind speed near the diffuser entrance. The model used was a circular diffuser with $L / D=1.5$ $(D=20 \mathrm{~cm})$. Circular brims of various heights of $h$ were attached to the diffuser exit. Figure 10 shows the wind velocity distribution $U / U_{\infty}$ (Fig. 10(a)) and the static pressure distribution $C_{\mathrm{p}}$ (Fig. 10(b)) on the central axis. It was found that a brim of around $h / D=0.25-0.38$ is the most effective for wind acceleration. From Fig. 10(b), a brim larger than $h / D=0.38$ causes a pressure increase in the upstream flow in front of the diffuser and prevents the approaching wind from smoothly flowing in.

\section{Demonstration Experiment of Wind Power Genera- tion Performance}

\subsection{Examination of the optimal brimmed diffuser form with a wind turbine installed}

Applying the brimmed diffuser model to a small-size wind turbine, we examined the power output increase compared to a standard wind turbine. Figure 11 shows the experimental arrangement. It needs to be made into a compact structure to some degree if commercialization is to be realized. We used a short circular diffuser of $L / D=1.25$ in this demonstration experiment. The entrance diameter was

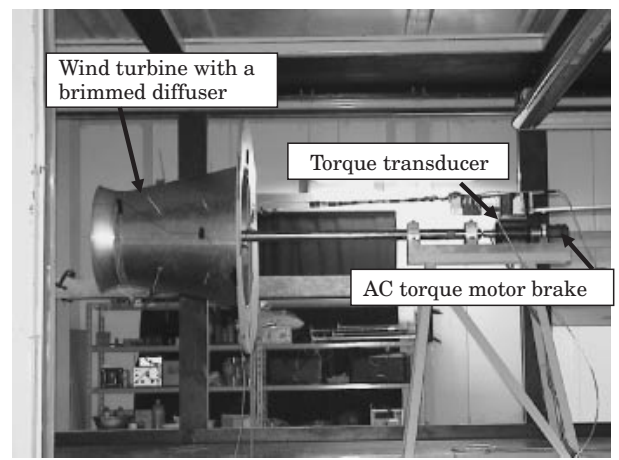

Fig. 11. Experiment on the power output generation of a wind turbine equipped with a brimmed diffuser shroud.

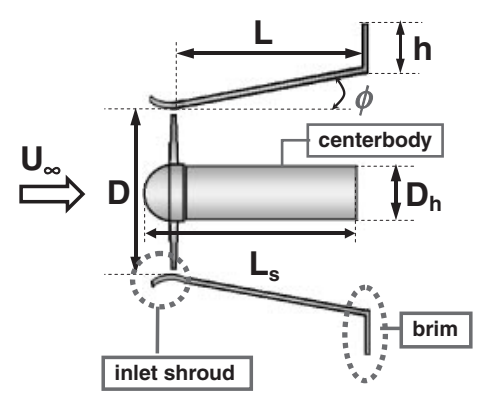

Fig. 12. Schematic of a wind turbine equipped with a brimmed diffuser shroud.

$D=60 \mathrm{~cm}$, and a brim height of $h / D=0.5$ was adopted. As shown in Fig. 10, the optimal size of the brim is $h / D=$ $0.25-0.38$ if a wind turbine is not installed inside the diffuser shroud. However, if a wind turbine is installed and works on the wind as a form of resistance, a larger brim of around $h / D=0.5$ was effective for collecting and drawing the wind into the diffuser shroud.

Similarly, the diffuser opening angle $\phi$ (see Fig. 12) was changed from $\phi=4^{\circ}$ to $\phi=12^{\circ}$. It was found that when a wind turbine is installed in the diffuser entrance, it plays the role of a resistance body and controls separation of the flow inside the diffuser. ${ }^{10)} \mathrm{A}$ numerical simulation also proved this. Abe and Ohya ${ }^{11)}$ prepared a resistance screen model for the diffuser entrance as the wind turbine model for their numerical model and examined how the flow inside the diffuser changes with the degree of the resistance screen. As a result, they showed that, in the case of the resistance model, the flow separation near the diffuser entrance can be suppressed as the wind flows along the inner wall of the diffuser. Thus, the installation of a wind turbine reduces wind energy and works as a resistance body to the wind. For another reason, it seems that the diffuser becomes more efficient due to the additional swirl mixing mechanism, which increases boundary layer momentum exchange. Therefore, this makes the adoption of a wider diffuser opening angle possible and leads to an improvement in the pressure recovery coefficient of the diffuser. This is an important parameter for shrouded wind turbines with brimmed diffusers. ${ }^{12)}$

Furthermore, we examined other shape parameters of the 


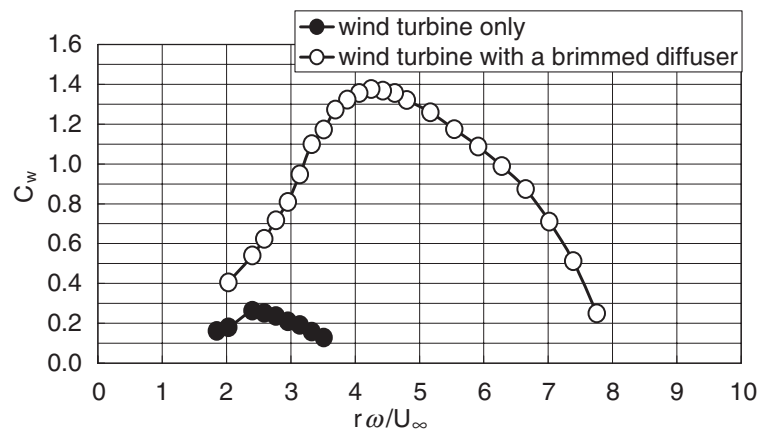

Fig. 13. The comparison of power coefficients between the wind turbine equipped with a brimmed diffuser shroud and a standard wind turbine.

brimmed diffuser such as the inlet shroud, hub ratio, centerbody length and so on, as illustrated in Fig. 12. Finally, we adopted a hub ratio of $D_{\mathrm{h}} / D=22 \%$ and centerbody length of $L_{\mathrm{S}}=0.75 L .^{10)}$ Thus, the optimal shape for the brimmed diffuser shroud used with a wind turbine has been found.

\subsection{Output performance test of a wind turbine with a brimmed diffuser shroud}

As for the experimental method, a torque transducer (the rating $0.5 \mathrm{~N} \cdot \mathrm{m}$ ) was connected to the wind turbine and an AC torque motor brake was set behind it for the loading. We measured the torque $Q(\mathrm{~N} \cdot \mathrm{m})$ and rotational speed $n$ $(\mathrm{Hz})$ of the wind turbine under the condition of gradually increasing turbine load from zero. The calculated power output $P(\mathrm{~W})=Q \cdot 2 \pi n$ is shown as a performance curve. A wind turbine with a brimmed diffuser shroud was supported by a long straight bar from the measurement bed, which was placed downstream and consisted of a torque transducer, a revolution sensor and an AC torque motor brake, as shown in Fig. 11. The approaching wind speed $U_{\infty}$ was $11 \mathrm{~m} / \mathrm{s}$, and the representative scale length equalled the diffuser entrance diameter $D(=60 \mathrm{~cm})$, giving a Reynolds number of $R e=4.5 \times 10^{5}$.

Figure 13 shows the experimental results. The horizontal axis shows the blade-tip speed ratio $\lambda=\omega r / U_{\infty}$. Here, $\omega$ is the angular frequency, $2 \pi n$, and $r$ is the radius of the wind turbine rotor. The vertical axis shows the power coefficient $C_{\mathrm{w}}\left(=P /\left(0.5 \rho U_{\infty}{ }^{3} A\right)\right.$; where, $A$ is the rotational area of the wind turbine rotor, $\left.\pi r^{2}\right)$. A wind turbine blade with a NACA63-2 wing section contour was designed using a three-bladed wind turbine, resulting in an optimum tip speed ratio of 5.0. As shown in Fig. 13, when a brimmed diffuser is applied, a remarkable increase in the output power coefficient of approximately five times that of a conventional wind turbine is achieved. The experimental results shown in Fig. 13 were obtained under the same wind speed and swept area of a wind turbine.

\section{Important Features of a Wind Turbine with Brim- med Diffuser Shroud}

Figure 14 shows the first prototype model of a wind turbine equipped with a brimmed diffuser shroud $(500 \mathrm{~W}$ class). The diffuser length of this model is 1.25 times as long

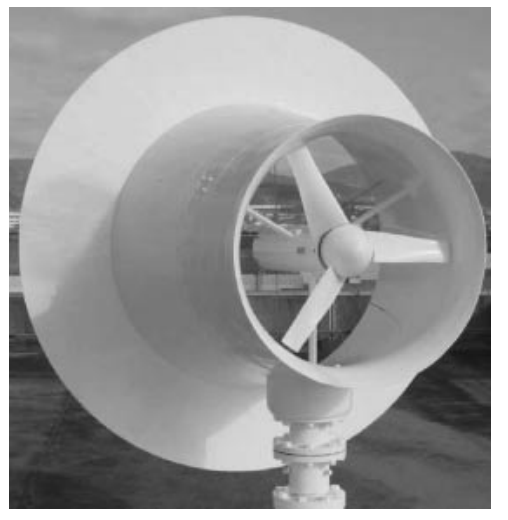

Fig. 14. Prototype wind turbine with a brimmed diffuser ( $500 \mathrm{~W}$ class).

as the diameter of the diffuser entrance $D(L=1.25 D$, $D=0.72 \mathrm{~m}$ ). The width of the brim $h$ is $0.5 D$. In a field experiment using the new wind turbine, the performance was as expected and equalled that of the wind tunnel experiment. The important features of the wind turbine equipped with a brimmed diffuser shroud are as follows:

1) Fivefold increase in output power as compared to conventional wind turbines due to concentration of the wind energy.

2) Brim-based yaw control: The brim at the exit of the diffuser makes wind turbines equipped with a brimmed diffuser rotate following the change in the wind direction, like a weathercock. As a result, the wind turbine automatically turns to face the wind.

3) Significant reduction in wind turbine noise: Basically, an aerofoil section of the turbine blade which gives the best performance in a low-tip speed ratio range is chosen. Since the vortices generated from the blade tips are considerably suppressed through the interference with the boundary layer within the diffuser shroud, the aerodynamic noise is reduced substantially.

4) Improved safety: The wind turbine, rotating at a high speed, is shrouded by a structure and is also safe against damage from broken blades.

\section{Conclusions}

We have developed a new wind turbine system that can generate power output under the condition of low wind speed and obtain a remarkably higher power output compared to conventional systems.

We examined a wind collection-acceleration device that makes it possible to concentrate the wind energy (i.e., increase the wind velocity locally and draw the wind to a wind turbine). It was confirmed that a hollow-structure diffuser is as effective as the shroud form for collecting and accelerating the wind.

To obtain a further increase in wind speed inside the diffuser shroud, a brim of proper height is attached to the outer periphery of the diffuser exit, successfully realizing a remarkable increase in wind speed of 1.6-2.4 times that of the approaching wind speed. This is because the brim 
generates a very-low-pressure region in the exit area of the diffuser by forming strong vortices and drawing the wind into the diffuser.

We conducted a demonstration experiment in which power was generated using the wind turbine equipped with a brimmed diffuser shroud. To obtain a higher power output from the shrouded wind turbine, we investigated the optimal form of the brimmed diffuser with a wind turbine installed. Namely, we examined various shape parameters of the brimmed diffuser such as the diffuser opening angle, brim height, hub ratio, centerbody length, inlet shroud and so on. As a result, the new wind turbine equipped with a brimmed diffuser shroud demonstrated power augmentation for a given turbine diameter and wind speed by a factor of about five compared to a standard wind turbine.

Incidentally, owing to the existence of the brim, the shrouded wind turbine rotates in the horizontal plane and always faces in the direction of the approaching wind. Thus, the yawing motion is automatically controlled by the brim. Furthermore, application of the diffuser shroud results in a significant reduction of wind turbine noise (blade-tip noise) and improves safety against broken blades.

\section{Acknowledgments}

This work was supported by four organizations including a Grant-in-Aid for Scientific Research, No. 14205139, sponsored by the Ministry of Education, Culture, Sports, Science and Technology, Japan, Environment Protection Research sponsored by the Sumitomo Fund, Fluid Machinery Research sponsored by the Harada Memorial Fund, and the Program and Project for Education and Research of Kyushu University. It was also supported by a matching fund from the Ministry of Economy, Trade and Industry. We gratefully acknowledge our laboratory staff, Messrs. N.
Fukamachi and K. Watanabe, Research Institute for Applied Mechanics, Kyushu University, for their great cooperation in the experiments and data analysis.

\section{References}

1) Gilbert, B. L., Oman, R. A. and Foreman, K. M.: Fluid Dynamics of Diffuser-Augmented Wind Turbines, J. Energy, 2 (1978), pp. 368374.

2) Gilbert, B. L. and Foreman, K. M.: Experiments with a DiffuserAugmented Model Wind Turbine, Trans. ASME, J. Energy Resources Technology, 105 (1983), pp. 46-53.

3) Nagai, M. and Irabu, K.: Momentum Theory for Diffuser Augmented Wind Turbine, Trans. JSME, 53 (1987), pp. 1543-1547 (in Japanese).

4) Phillips, D. G., Richards, P. J. and Flay, G. J.: CFD Modelling and the Development of the Diffuser Augmented Wind Turbine, Proceedings of the Comp. Wind Eng. 2000, Birmingham, 2000, pp. 189-192.

5) Nemoto, Y., Anzai, A. and Ushiyama, I.: A Study for the Concentrator Augmented Wind Turbine, Wind Energy, 27, 2 (2003), pp. 51-55 (in Japanese).

6) Ushiyama, I.: Introduction of Wind Turbine, Sanseido Press, Tokyo, 1997, pp. 77-84 (in Japanese).

7) Suito, H. and Ishii, K.: Simulation of Dynamic Stall by MultiDirectional Finite-Difference Method, Proceedings of the 26th AIAA Fluids Dynamics Conference, 1995, pp. 1-8.

8) Karasudani, T., Ohya, Y., Fukamachi, N. and Watanabe, K.: WindVelocity Acceleration by Hollow Bodies, J. Jpn. Soc. Fluid Mech., Nagare, 22 (2003), pp. 337-343 (in Japanese).

9) Ohya, Y., Karasudani, T. and Sakurai, A.: Development of HighPerformance Wind Turbine with a Brimmed Diffuser, J. Jpn. Soc. Aeronaut. Space Sci., 50 (2002), pp. 477-482 (in Japanese).

10) Ohya, Y., Karasudani, T. and Sakurai, A.: Development of HighPerformance Wind Turbine with a Brimmed Diffuser-Part 2-, J. Jpn. Soc. Aeronaut. Space Sci., 52 (2004), pp. 210-213 (in Japanese).

11) Abe, K. and Ohya, Y.: An Investigation of Flow Fields around Flanged Diffusers Using CFD, J. Wind Eng. Ind. Aerodyn., 92 (2004), pp. 315-330.

12) Inoue, M., Sakurai, A. and Ohya, Y.: A Simple Theory of Wind Turbine with a Brimmed Diffuser, Turbomachinery, 30 (2002), pp. 497-502 (in Japanese). 